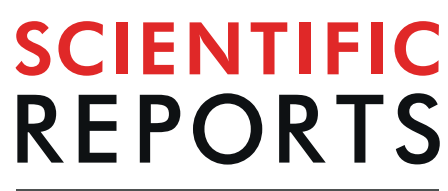

natureresearch

Received: 28 August 2018

Accepted: 7 August 2019

Published online: 22 August 2019

\section{Mean diffusivity related to rule- breaking guilt: the Macbeth effect in the sensorimotor regions}

Seishu Nakagawa ${ }^{1,2}$, Hikaru Takeuchi ${ }^{3}$, Yasuyuki Taki ${ }^{3,4,5}$, Rui Nouchi ${ }^{6,7}$, Yuka Kotozaki ${ }^{7}$, Takamitsu Shinada ${ }^{2}$, Tsukasa Maruyama ${ }^{2}$, Atsushi Sekiguchi ${ }^{4,8}$, Kunio lizuka $^{9}$, RyoichiYokoyama ${ }^{10}$, Yuki Yamamoto ${ }^{2}$, Sugiko Hanawa ${ }^{2}$, Tsuyoshi Araki ${ }^{11}$, Carlos Makoto Miyauchi ${ }^{2,12}$, Daniele Magistro ${ }^{13}{ }^{13}$, Kohei Sakaki ${ }^{14}$, Hyeonjeong Jeong ${ }^{2,15}$, Yukako Sasaki ${ }^{14}$ \& Ryuta Kawashima ${ }^{3,7,14}$

Guilt, a self-conscious emotion, includes self-focused role taking and also correlates with other-oriented role-taking. Excess guilt proneness might be relevant to obsessive compulsive disorders. The white matter (WM) neural correlates of the degree of guilt have not yet been determined. We hypothesized that the WM structures involved in feelings of guilt are associated with social and moral cognition (inferior parietal lobule [IPL], prefrontal cortex [PFC], and cingulate), and aimed to visualize this using diffusion MRI. We investigated the association between regional WM structures (WM volume, and fractional anisotropy, and mean diffusivity [MD]), and feelings of guilt in 1196 healthy, young students using MRI and the Guilty Feeling Scale, which comprises interpersonal situation (IPS; guilt from hurting friends) and rule-breaking situation (RBS; deontological guilt) scores. The primary novel finding presented here is that MD in the right somatosensory and motor cortices from arm to hand were positively correlated with RBS scores. Further, consistent with our hypothesis, RBS scores were positively correlated with MD in the same regions. These results would be predicted by the Macbeth effect, an obsession with dirt leading to hand-washing rituals resulting from guilt, made famous by the Shakespearian character Lady Macbeth. "What, will these hands ne'er be clean?" William Shakespeare (Shakespeare, 1606) Macbeth.

Guilt, a dysphoric feeling, involves one's negative evaluation of specific behaviours or transgression ${ }^{1}$. Moreover, guilt is a self-conscious emotion, and self-reflection is necessary to induce guilt. Contrary to this self-oriented emotion, guilt-proneness is also correlated with other-oriented perspective-taking and empathic concern ${ }^{2}$. In brief, self-focused and other-focused role-taking might be useful for understanding the classification of guilt. People imagine themselves in others' situation as self-focused role-taking, whereas they focus directly on others' distress as other-focused role-taking ${ }^{3}$.

${ }^{1}$ Division of Psychiatry, Tohoku Medical and Pharmaceutical University, Sendai, Japan. ${ }^{2}$ Department of Human Brain Science, Institute of Development, Ageing and Cancer, Tohoku University, Sendai, Japan. ${ }^{3}$ Division of Developmental Cognitive Neuroscience, Institute of Development, Ageing and Cancer, Tohoku University, Sendai, Japan. ${ }^{4}$ Division of Medical Neuroimaging Analysis, Department of Community Medical Supports, Tohoku Medical Megabank Organization, Tohoku University, Sendai, Japan. ${ }^{5}$ Department of Nuclear Medicine and Radiology, Institute of Development, Ageing and Cancer, Tohoku University, Sendai, Japan. ${ }^{6} \mathrm{Creative} \mathrm{Interdisciplinary} \mathrm{Research}$ Division, Frontier Research Institute for Interdisciplinary Science (FRIS), Tohoku University, Sendai, Japan. ${ }^{7}$ Smart Ageing International Research Center, Institute of Development, Ageing and Cancer, Tohoku University, Sendai, Japan. ${ }^{8}$ Department of Behavioral Medicine, National Institute of Mental Health, National Center of Neurology and Psychiatry, Kodaira, Tokyo, Japan. ${ }^{9}$ Department of Psychiatry, Tohoku University Graduate School of Medicine, Sendai, Japan. ${ }^{10}$ School of Medicine, Kobe University, Kobe, Japan. ${ }^{11}$ ADVANTAGE Risk Management Co., Ltd, Tokyo, Japan. ${ }^{12}$ Department of Language Sciences, Graduate School of Humanities, Tokyo Metropolitan University, Tokyo, Japan. ${ }^{13}$ Department of Sport Science, School of Science and Technology, Nottingham Trent University, Nottingham, United Kingdom. ${ }^{14}$ Advanced Brain Science, Institute of Development, Aging and Cancer, Tohoku University, Sendai, Japan. ${ }^{15} \mathrm{Graduate}$ School of International Cultural Studies, Tohoku University, Sendai, Japan. Correspondence and requests for materials should be addressed to S.N. (email: seishu.nakagawa.e8@tohoku.ac.jp) 


\begin{tabular}{|l|l|l|l|l|l|}
\hline Measure & Total & $\begin{array}{l}\text { Males } \\
(\boldsymbol{N}=\mathbf{6 8 6})\end{array}$ & $\begin{array}{l}\text { Females } \\
(\boldsymbol{N}=\mathbf{5 1 0})\end{array}$ & $\boldsymbol{P}$ & $\boldsymbol{F}$ \\
\hline Age & $20.7(1.8)$ & $20.8(1.9)$ & $20.6(1.6)$ & 0.051 & 3.8 \\
\hline RAPM & $28.5(3.9)$ & $28.8(3.9)$ & $28.1(3.8)$ & $0.002^{*}$ & 9.2 \\
\hline IPS guilt & $34.0(5.2)$ & $32.9(5.1)$ & $35.6(5.0)$ & $<0.001^{* *}$ & 85.5 \\
\hline RBS guilt & $21.1(5.0)$ & $20.1(4.8)$ & $22.4(5.0)$ & $<0.001^{* *}$ & 65.8 \\
\hline
\end{tabular}

Table 1. Sex differences in age, the RAPM, and IPS and RBS guilt scores (mean \pm SD): one-way ANOVA results. $* P<0.01, * * P<0.001$. Abbreviations: ANOVA, analysis of variance; IPS, interpersonal situation; RAPM, Raven's Advanced Progressive Matrix; RBS, rule-breaking situation; SD, standard deviation.

Deontological guilt arises from a self-judgement of having transgressed social and moral norms. Furthermore, deontological guilt motivates behaviour that is adapted to social and cultural rules ${ }^{4}$.

As for brain studies, a systematic review of 16 functional magnetic resonance imaging (fMRI) studies revealed guilt-related clusters of brain activation (voxel-based false discovery rate [FDR] corrected $P<0.05$ ) located in the prefrontal, temporal, and parietal regions, mainly in the left hemisphere and the left dorsal cingulate cluster (voxel-based family wise error [FWE] correction $P<0.05)^{5}$. Another systematic review of 21 functional and structural imaging studies demonstrated that guilt was more likely to be associated with activity in ventral anterior cingulate cortex, posterior temporal regions, and the precuneus than were other negative moral emotions (e.g. shame and embarrassment) ${ }^{6}$. A previous brain grey matter (GM) anatomical study conducted by our group directly demonstrated that scores related to feelings of guilt in both interpersonal and rule-breaking situations were uniquely and negatively related to regional GM density (rGMD) in the right posterior insula (PI) ${ }^{7}$. To the best of our knowledge, no studies have used diffusion tensor imaging (DTI) to assess the correlation of guilt feelings and white-matter (WM) structure, including WM volume (WMV).

From a social-cognitive perspective, social knowledge is represented in the medial prefrontal cortex $(\mathrm{mPFC})^{8}$. The social identity representing values in the social context was based on the ventral MPFC (vmPFC) and the posterior cingulate cortex 9 . Further, the orbitofrontal cortex (OFC) plays a known role in executive function and in controlling and correcting punishment-related behaviour ${ }^{10}$. Moreover, morally-relevant information is processed in the inferior parietal lobule (IPL), including the superior temporal sulcus (STS), inferior frontal gyrus (IFG), and dorsolateral PFC (DLPFC) ${ }^{11}$.

As we have described previously ${ }^{12}$, diffusion MRI is well suited to studies of WM development because changes in diffusion MRI signals may reflect alterations in myelination, a form of axonal packing that occurs with age, and may be associated with changes in emotion and cognition ${ }^{13}$. These tissue changes are thought to affect neural plasticity ${ }^{14}$. Most significantly, it has been suggested that mean diffusivity (MD), rather than radial and axial diffusivity, indicates neural plasticity ${ }^{14}$. For more details, please refer to the studies ${ }^{13,14}$.

Accordingly, we hypothesized that the WM structures that underlie feelings of guilt are also involved in social and moral cognition (IPL including STS, and PFC [MPFC, OFC, IFG, and DLPFC]). We also hypothesized that we could identify regions sensitive to the development of these capacities in young adults using diffusion MRI. Given this, the purpose of this study was to identify the WM structure associated with two types of feelings of guilt often experienced by young people. We further investigated whether the relationship between WM structures and guilt scores differed between males and females using a whole-brain level analysis, because females are often more willing to concede responsibility for misdeeds and may have more difficulty in getting rid of feelings of guilt than males ${ }^{15}$.

\section{Results}

Behavioural data. Table 1 shows the means and standard deviations (SD) for age and the scores on the Raven's Advanced Progressive Matrix (RAPM), and guilty feeling. Figure 1 depicts the distributions of the interpersonal situation (IPS) and rule-breaking situation (RBS) scores in males and females. There were significant differences between males and females in the RAPM, IPS, and RBS scores $(P<0.01$, one-way analysis of variance [ANOVA]).

MRI data. Analysis of voxel-based morphometry (VBM) data. After controlling for sex, age, and RAPM scores, there were no significant positive or negative correlations between IPS or RBS scores and regional WMV (rWMV) at each voxel at a FWE-corrected threshold of $P<0.05$, based on the threshold-free cluster enhancement (TFCE) method at the level of the whole brain.

Analysis of fractional anisotropy (FA) and MD data. A whole-brain multiple regression analysis that was performed after controlling for sex, age, total intracranial brain volume (TIV; total GM volume + total WMV + total cerebrospinal fluid [CSF] volume), and RAPM scores revealed no significant correlation between RBS scores (but not IPS scores) and FA.

We found a significant correlation between the IPS (or RBS) scores and MD using the same analyses described above in the bilateral inferior parietal lobule (IPL), the right prefrontal region in the centre of middle frontal region (from ventral to dorsal and medial to lateral regions including insula) and post- and precentral regions, and the left posterior cingulate region, using the TFCE method with FWE corrected to $P<0.0125(0.05 / 4)$ at the whole-brain level (Table 2 and Figure 2). 
A

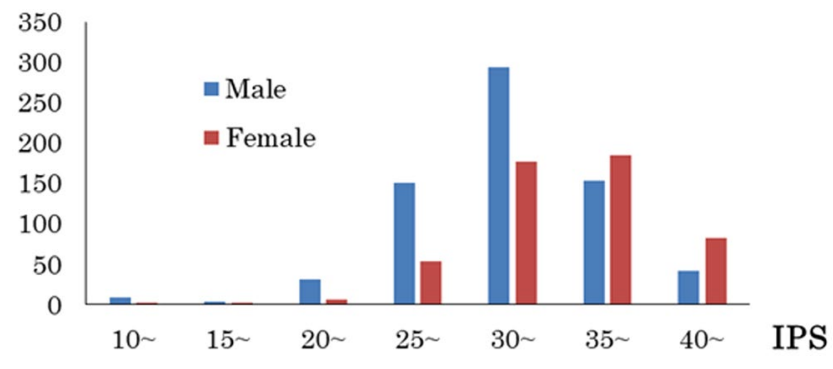

N B

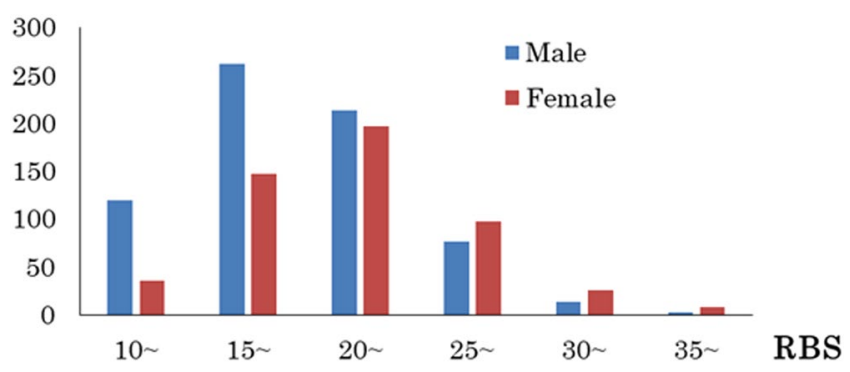

Figure 1. (A) Distribution of interpersonal situation (IPS) scores in males and females. (B) Distribution of rule-breaking situation (RBS) scores in males and females. Histograms show the distributions of IPS/RBS scores in males and females.

\begin{tabular}{|c|c|c|c|c|c|c|c|c|}
\hline Brain region & $\mathbf{R} / \mathbf{L}$ & $\mathbf{x}$ & $\mathbf{y}$ & $\mathbf{z}$ & \begin{tabular}{|l|} 
TFCE \\
Value \\
\end{tabular} & $\begin{array}{l}\text { Corrected } P \text {-value } \\
(\text { FWE })\end{array}$ & $\begin{array}{l}\text { Cluster } \\
\text { size }\left(k_{\mathrm{E}}\right)\end{array}$ & $\beta$ \\
\hline Precentral region & \multirow{3}{*}{$\mathrm{R}$} & 21 & -25.5 & 63 & 1185.5 & $0.011^{*}$ & \multirow{3}{*}{416} & \multirow{3}{*}{0.099} \\
\hline Postcentral region & & 10.5 & -28.5 & 75 & 1169.6 & $0.011^{*}$ & & \\
\hline Precentral region & & 18 & -19.5 & 51 & 1148.7 & $0.012^{*}$ & & \\
\hline Middle frontal region & \multirow{3}{*}{$\mathrm{R}$} & 27 & 22.5 & 28.5 & 1816.4 & $0.005^{*}$ & \multirow{3}{*}{11549} & \multirow{3}{*}{0.113} \\
\hline Operculum part of IFR & & 39 & 21 & 16.5 & 1788.8 & $0.005^{*}$ & & \\
\hline Middle frontal region & & 22.5 & 40.5 & 13.5 & 1776.9 & $0.005^{*}$ & & \\
\hline Precuneus & \multirow{2}{*}{$R$} & 27 & -48 & 28.5 & 1396.5 & $0.009^{*}$ & \multirow{2}{*}{1071} & \multirow{2}{*}{0.125} \\
\hline Parietal operculum & & 46.5 & -33 & 30 & 1149.6 & $0.012^{*}$ & & \\
\hline Superior parietal lobule & \multirow{3}{*}{$\mathrm{L}$} & -22.5 & -42 & 33 & 1264.7 & $0.011^{*}$ & \multirow{3}{*}{1166} & \multirow{3}{*}{0.113} \\
\hline Precuneus & & -15 & -43.5 & 39 & 1206.9 & $0.011^{*}$ & & \\
\hline Precuneus & & -25.5 & -61.5 & 12 & 1175.1 & $0.011^{*}$ & & \\
\hline Parietal operculum & \multirow{2}{*}{$\mathrm{L}$} & -45 & -24 & 25.5 & 1162.1 & $0.011^{*}$ & \multirow{2}{*}{349} & \multirow{2}{*}{0.091} \\
\hline Central operculum & & -43.5 & -15 & 24 & 1158.5 & $0.011^{*}$ & & \\
\hline posterior cingulate region & $\mathrm{L}$ & -19.5 & -54 & 7.5 & 1133.0 & $0.011^{*}$ & 1 & 0.087 \\
\hline
\end{tabular}

Table 2. Brain regions exhibiting a significant correlation between $\mathrm{MD}$ and rule-breaking situation scores. $* P<0.0125$, FWE corrected. FWE: family-wise errors, IFR: inferior frontal region; IPL: inferior parietal lobule, L: left, R: right, MD: Mean diffusivity, TFCE: threshold-free cluster enhancement.

Interaction effects of sex and IPS or RBS on brain structures. Using data from both sexes with respect to the covariates of age, RAPM, TIV, and IPS (or RBS) scores, an analysis of covariance (ANCOVA) revealed no significant interaction effect between IPS (or RBS) scores and sex on rWMV, FA, or MD using the TFCE method with FWE corrected to $P<0.05$ at the whole-brain level.

\section{Discussion}

The present study is the first to investigate direct associations between feelings of guilt and white matter structures in healthy individuals at the whole-brain level. Measures of guilt can be classified into two categories: assessment of states and traits ${ }^{16}$. RBS guilt and IPS guilt seem to be more characteristic traits of guilt rather than the state of guilt using the participant's answers to questionnaires in many different situations. Importantly and unexpectedly, the right post- and precentral regions were found to be neural correlates of RBS. Consistent with our hypothesized neural correlates for social and moral cognition (IPL including STS and PFC [mPFC, OFC, IFG, and 
A.1
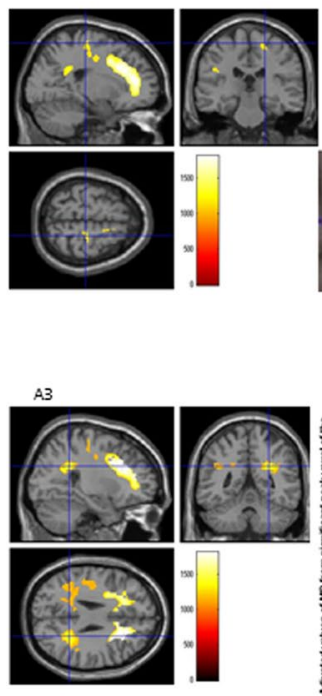

B1

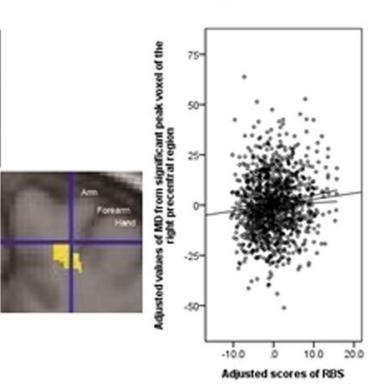

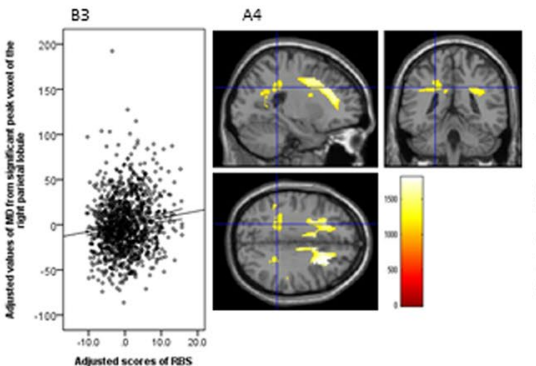
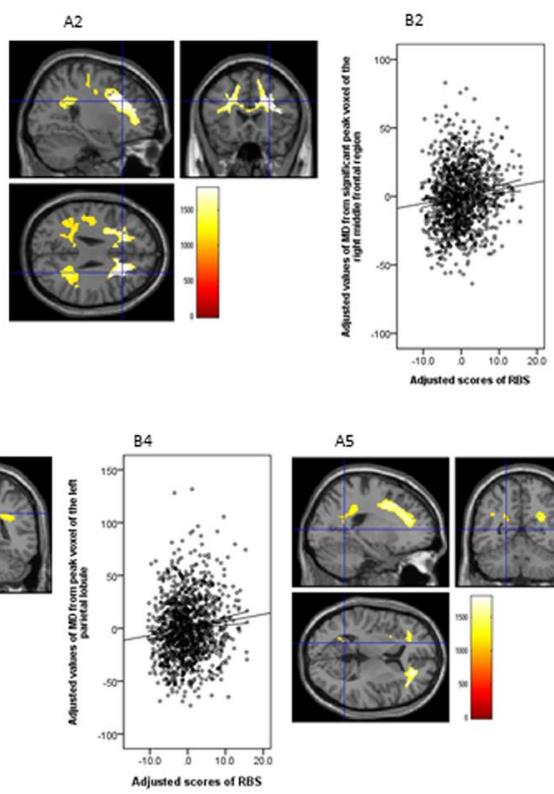

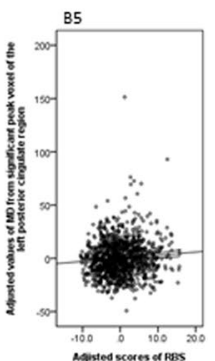

Figure 2. Regions correlated with mean diffusivity (MD) and rule-breaking situation (RBS) scores. The present results were determined based on a family-wise error (FWE)-corrected threshold of $P<0.0125(0.05 / 4)$ with a threshold-free cluster enhancement (TFCE) based on 5000 permutations; the results were corrected at the whole-brain level. Regions showing correlations were overlaid onto a single T1 image in the SPM8 toolbox. The red-to-yellow colour scale indicates the level of the TFCE value's positive correlation with the MD and RBS scores; areas with significant correlations were identified in the right pre and postcentral regions (A1), prefrontal region in the centre of middle frontal region (widespread from medial to lateral and from ventral to dorsal prefrontal regions including the insula) (A2), parietal lobule (A3), and the left parietal lobule including the inferior parietal lobule (IPL) (A4) and the posterior cingulate region (A5). Residual plots with trend lines depicting correlations between residuals in the multiple regression analyses with RBS scores as the dependent variable and other confounding factors as independent variables; 95\% confidence intervals for the trend lines are shown. The peak regional MD values in the key regions in the right precentral region (B1), middle frontal region (B2), IPL (B3), the left parietal lobule (B4), and the posterior cingulate region (B5) are depicted.

DLPFC]), total RBS scores were positively associated with MD values in the bilateral IPL and the right prefrontal region (widespread from ventral to dorsal and medial to lateral regions including the insula), as well as the left posterior cingulate region.

First, we should outline potential mechanisms by which the post- and precentral regions (sensorimotor regions from arm to hand $)^{17}$ relate to RBS scores. Involvement of the sensorimotor regions in the embodied metaphor of moral-purity is somatotopically organized by linking moral purity with cleansing such as the hands and mouth ${ }^{18,19}$. The 'Macbeth effect' addresses a threat to one's moral purity by cleansing the physical body, especially the hands ${ }^{20}$. Physical cleansing is a psychological mechanism for addressing moral emotions including guilt ${ }^{20}$. Further, the somatosensory cortex is also relevant to the somatic maker hypothesis, which is relevant to the body state structure when the awareness of perceptual signals is necessary for regulating emotions ${ }^{21}$.

Second, there were no significant associations between RBS and other WM measures (rWMV and FA). A plausible reason for this is that DTI results are particularly influenced by WM maturation during adolescence ${ }^{22}$. Young adults in this study might demonstrate higher RBS scores due to developmentally-delayed WM maturation after adolescence. As shown in our previous study ${ }^{12}, \mathrm{MD}$ and FA measure different microstructural brain properties. MD assesses capillaries, spines, macromolecular proteins, myelin properties, membranes, axons, neuron shapes, protoplasmic and fibrous glia, and enhanced tissue organization ${ }^{23}$. FA is more strongly associated with microstructural properties related to brain connectivity, and is sensitive to increases in axonal membrane thickness, diameter, and/or the extent of parallel organization in axons ${ }^{23}$. Further, FA values are more stable over time (from adolescence to middle age) than $M D$ values ${ }^{24}$. This difference in the degree of change over time might affect sensitivity of FA and MD to detecting regions associated with guilt ${ }^{12}$. For more details, please refer to our previous study ${ }^{12}$.

Third, we should discuss the detected regions in terms of our hypothesis. RBS guilt is more self-oriented than IPS guilt. Notably, the brain's default mode network (DMN) includes the detected regions, namely the mPFC, PCC, IPL, and precuneus ${ }^{25}$. The DMN, which shows higher levels of activity during passive task conditions than during goal-directed task conditions ${ }^{25}$, has become almost synonymous with self-referential mental activity $^{26}$ in order to meet social cognitive demands ${ }^{27}$. With respect to the PFC, the mPFC is important for reflective self-processing ${ }^{28}$. Guilt has been shown to recruit greater prefrontal regions, a process which may represent demands for behavioural change ${ }^{29}$. Deontological guilt emerges from the internal understanding that one has violated an intuitive moral rule. Punishment is an essential ingredient in deontological guilt because rank differences between people and authority are restored through punishment of the transgressor ${ }^{30}$. The right DLPFC plays a 
key role in third-party punishment ${ }^{31}$. Based on the somatic marker hypothesis, the vmPFC has an important role in the processes of reasoning and decision making ${ }^{21}$. Defective somatic markers in the vmPFC may serve as a neural framework for explaining immoral or corrupt behaviours ${ }^{32}$. Thus, the vmPFC has an essential role for integrating emotional signals in complex social situations ${ }^{33}$. Further, activity in the vmPFC could link affective disorders to regulation of stressful environments ${ }^{34}$. As for the cingulate cortex, deontological (rule-breaking) moral judgements led to increased activation in the PCC and the right temporoparietal junction ${ }^{35}$. Activity in the PCC was associated with self-relevant moral dilemmas ${ }^{36}$. As we have described previously ${ }^{37}$, regions from the anterior cingulate region to the anterior insula form a salience network, responsible for integrating interceptive information with emotional salience, as in social anxiety. Moreover, deontological guilt selectively activates the insula ${ }^{38}$ and is involved in the processing of disgust and self-reproach ${ }^{30}$. Further, the insula has been shown to encode cognition related to inequity when considering ethical fairness ${ }^{39}$. As described in the introduction, moral reasoning involves a diversity of brain areas including the IPL ${ }^{11}$. The IPL might form a cognitive hub that is uniquely human, and multiple networks converge and interact in the IPL ${ }^{40}$. Interestingly, deontological guilt is involved in obsessive-compulsive disorder (OCD), which is often associated with decreased activation of the ACC and the insula in a clinical fMRI study ${ }^{41}$. Exaggerated guilt-proneness might be relevant to clinical thought-related pathology, such as depression, obsessive compulsive disorders, and paranoia ${ }^{42}$. Greater deontological guilt was related to higher MD (a lower density of neural tissues prohibits free water diffusion), a finding that might relate to diminished moral and social cognition. Decrements to the above-mentioned functions seem to be fundamental of the feeling of deontological guilt.

Fourth, both IPS and RBS scores were higher in females than in males, although no significant gender difference related to the scores was found in regional brain structures. The psychological results were in accordance with the widespread view that women in adolescence through adulthood experience more guilt than men ${ }^{43}$. However, gender differences in guilt feelings tend to be larger among whites than people of other races, in a meta-analysis found ${ }^{43}$. A pilot fMRI study examining neural underpinnings of guilt in a small sample of Germans showed that women activated only temporal regions when experiencing guilt, whereas men showed additional frontal, occipital, and amygdala activation ${ }^{44}$. Further, evidence of gender difference in moral orientation seems to be weak and inconsistent ${ }^{45}$. A study on trait guilt using Japanese undergraduates found no significant gender differences ${ }^{46}$. Thus, the lack of gender differences in neural correlates of guilt feelings might be due to the weak effects of gender differences in morality itself, particularly among individuals who are not white ${ }^{43}$.

Finally, as we have previously noted ${ }^{47,48}$, there are a few limitations to the present study that should be mentioned. Because the present study used a cross-sectional design, the results presented here cannot be used to determine causality between RBS scores and various, associated brain regions. To overcome this limitation, a prospective study that confirms these brain regions are causally necessary to these functions (e.g. a lesion study) remains necessary. Furthermore, we used young, highly-educated, healthy subjects, which may affect generalisability of our findings.

\section{Methods}

Subjects. The present study included 1196 healthy, right-handed individuals (686 males and 510 females) with a mean age of $20.7 \pm 1.8$ years. Written informed consent was obtained from each participant prior to beginning the study in accordance with the Declaration of Helsinki (1991). All study procedures were approved by the Ethics Committee of Tohoku University. All experiments were performed in accordance with approved guidelines. For more details regarding procedures undertaken in the present study, please refer to our previous work ${ }^{49-51}$.

Psychological outcome measures. Assessment of feelings of guilt. We used the reliable and valid guilty feeling scale (GFS) ${ }^{52}$, which consists of IPS (11 items) and RBS (10 items) tests ${ }^{52}$. The GFS consisted of 21 statements in total, which were divided into two dimensions. We asked the participants to describe their anticipated feelings towards situations that often elicit feelings of guilt. The four-point rating scale ranged from 1 (no feeling) to 4 (extreme feeling). The internal consistencies (Cronbach's coefficient of alpha) of the IPS and RBS tests were 0.89 and $0.86^{52}$, respectively. Convergent validity for the scale was confirmed by significant correlations with other measures (empathy and role-taking ability [social perspective-taking]) related to feelings of guilt. IPS guilt mainly refers to situations in which a friend is hurt, whereas RBS guilt is more public and sometimes involves specific others (parents). For more details, please refer to our previous study ${ }^{7}$.

Psychometric measures of general intelligence. The RAPM, which is a widely used measure of general intelligence $^{53}$, was utilized in the present study. This measure was adjusted to assess the effects of general intelligence on brain structures ${ }^{54-56}$

Behavioural data analyses. All behavioural data were analysed using the IBM SPSS Statistics 22.0 software package (IBM Corp.; Armonk, NY, USA). Differences between males and females in terms of age and scores on the cognitive measures (RAPM, the IPS, and the RBS scores) were analysed by one-way ANOVA; a two-tailed $P$ value $<0.05$ was considered to indicate statistical significance.

Image acquisition. Structural MRI. All MRI data were acquired using a 3T Philips Achieva scanner (Philips Medical Systems, Best, Netherlands). Three-dimensional high-resolution T1-weighted images were collected using a magnetisation-prepared rapid gradient-echo sequence with the following parameters: $240 \times 240$ matrix, $\mathrm{TR}=6.5 \mathrm{~ms}, \mathrm{TE}=3 \mathrm{~ms}, \mathrm{TI}=711 \mathrm{~ms}, \mathrm{FOV}=24 \mathrm{~cm}, 162$ slices, in plane resolution $=1.0 \times 1.0 \mathrm{~mm}$, slice thickness $=1.0 \mathrm{~mm}$, and a scan duration of $483 \mathrm{~s}$. 
Diffusion-weighted data were acquired using a spin-echo echo-planar imaging (EPI) sequence with the following parameters: $\mathrm{TR}=10293 \mathrm{~ms}, \mathrm{TE}=55 \mathrm{~ms}$, FOV $=22.4 \mathrm{~cm}, 2 \times 2 \times 2 \mathrm{~mm}^{3}$ voxels, 60 slices, SENSE reduction factor $=2$, and number of acquisitions $=1$. Diffusion weighting was isotropically distributed in 32 directions $\left(b\right.$ value $\left.=1,000 \mathrm{~s} / \mathrm{mm}^{2}\right)$ and three images with no diffusion weighting ( $b$ value $=0 \mathrm{~s} / \mathrm{mm}^{2} ; \mathrm{b}=0$ images) were acquired using the spin-echo EPI sequence $\left(\mathrm{TR}=10293 \mathrm{~ms}, \mathrm{TE}=55 \mathrm{~ms}, \mathrm{FOV}=22.4 \mathrm{~cm}, 2 \times 2 \times 2-\mathrm{mm}^{3}\right.$ voxels, 60 slices). Acquisitions for phase correction and signal stabilization were performed, though these data were not used for image reconstruction. For more details regarding these procedures, please refer to our previous work $^{23,47,57}$

Pre-processing and analyses of structural data. VBM data. All pre-processing of the T1WIs data was performed using Statistical Parametric Mapping software (SPM8; Wellcome Department of Cognitive Neurology, London, UK) according to the protocol described for VBM analyses in a previous report from our group $^{58}$. Using the new segmentation algorithm implemented in SPM8, T1-weighted structural images from each individual were segmented and normalized to Montreal Neurological Institute (MNI) space to yield images with $1.5 \times 1.5 \times 1.5-\mathrm{mm}$ voxels using diffeomorphic anatomical registration through the exponentiated lie algebra (DARTEL) registration process implemented in SPM8. In addition, we performed a post-hoc volume change correction (modulation) ${ }^{59}$. All images were smoothed by convolving them using an isotropic Gaussian kernel of $6 \mathrm{~mm}$ full-width at half maximum (FWHM). For additional details, please refer to our previous work ${ }^{58,60}$.

FA and MD data. All pre-processing and analyses of the imaging data were performed using SPM8 implemented in MATLAB (MathWorks Inc.; Natick, MA, USA). The methods and parameters were optimized and validated using SPM8, whereas those using SPM12 were not validated in our previous study ${ }^{47}$. Normalized FA images were then masked by a custom mask image most likely to be white matter, then smoothed by a Gaussian kernel of $8 \mathrm{~mm}$ FWHM. These methods were adapted from a previous study ${ }^{37}$.

The MD map was calculated from the collected images using a commercially available diffusion tensor analysis package (Philips Medical Systems, Best, Netherlands), run on the MR console. These procedures involved corrections for motion and distortion caused by eddy currents, and all calculations were performed using a previously described method ${ }^{61}$. Briefly, the MD images from participants were normalized using a previously validated DARTEL-based registration process to develop images with $1.5 \times 1.5 \times 1.5 \mathrm{~mm}$ voxels ${ }^{61}$. Next, tissues that were least likely to be grey or white matter were manually removed and the images were smoothed by convolving them using an isotropic Gaussian kernel of 8-mm FWHM. For additional details, please refer to our previous work ${ }^{47,48}$.

As in our previous study ${ }^{47}$, we did not and could not utilize tract-based spatial statistics (TBSS $)^{62}$. We instead used SPM-based analyses and believe that our new DTI preprocessing methods substantially alleviate the problems associated with voxel-based DTI analyses ${ }^{62}$. Further, TBSS uses only the skeleton of an FA map, with the result that voxels containing peripheral white matter are not included. Hence, methodological improvements to TBSS are needed to increase its reliability ${ }^{63}$. For more details, please refer to our previous study ${ }^{47}$.

Statistical group-level analyses of imaging and behavioural data. Correction for multiple comparisons was performed using TFCE ${ }^{64}$ with randomised (5,000 permutations) nonparametric testing in the TFCE toolbox (http://dbm.neuro.uni-jena.de/tfce/). A FWE corrected threshold of $P<0.0125(0.05 / 4)$ was applied. For more details, please refer to a previous study by our group ${ }^{37}$.

VBM data. A whole-brain multiple regression analysis was performed in SPM8 and used to assess the association between rWMV and IPS (or RBS) scores. Covariates for this analysis included sex, age, RAPM score, and TIV. For each covariate, the overall mean was used for mean centring. Analyses for rWMV were performed in voxels for all subjects that showed a signal intensity $>0.05$.

We also investigated whether the relationship between rWMV and guilt scores differed between males and females at the level of the whole brain. We employed a voxel-wise ANCOVA in which sex difference was a group factor, using $t$-contrasts (using the full factorial option of SPM8). In this analysis, age, RAPM, TIV, and IPS (or RBS) scores were modelled to enable detection of unique relationships with rWMV (using the interaction option in SPM8 for each sex). TIV was modelled to have a common relationship with rWMV across both sexes.

FA and MDdata. A voxel-by-voxel regression analysis was performed in SPM8 using the FA or MD value at each voxel as the dependent variable and age, sex, RAPM score, and IPS (or RBS) scores as independent variables. The analysis using FA was limited to areas within the white matter, whereas the analysis using MD was limited to areas within the grey and white matter masks that were created using the procedures described above. We also investigated whether the relationship between FA or MD and IPS (or RBS) scores differed between men and women by using the same method as in rWMV.

\section{References}

1. Niedenthal, P. M., Tangney, J. P. \& Gavanski, I. "If only I weren't" versus "if only I hadn't": distinguishing shame and guilt in counterfactual thinking. J Pers Soc Psychol 67, 585-595 (1994).

2. Tangney, J. P., Stuewig, J. \& Mashek, D. J. Moral emotions and moral behavior. Annual Review of Psychology 58, 345-372, https://doi. org/10.1146/annurev.psych.56.091103.070145 (2007).

3. Hoffman, M. L. Empathy and moral development. The Annual Report of Educational Psychology in Japan 35, 157-162 (1996).

4. Carni, S., Petrocchi, N., Del Miglio, C., Mancini, F. \& Couyoumdjian, A. Intrapsychic and interpersonal guilt: a critical review of the recent literature. Cognitive processing 14, 333-346, https://doi.org/10.1007/s10339-013-0570-4 (2013).

5. Gifuni, A. J., Kendal, A. \& Jollant, F. Neural mapping of guilt: a quantitative meta-analysis of functional imaging studies. Brain imaging and behavior 11, 1164-1178, https://doi.org/10.1007/s11682-016-9606-6 (2017).

6. Bastin, C., Harrison, B. J., Davey, C. G., Moll, J. \& Whittle, S. Feelings of shame, embarrassment and guilt and their neural correlates: A systematic review. Neurosci Biobehav Rev 71, 455-471, https://doi.org/10.1016/j.neubiorev.2016.09.019 (2016). 
7. Nakagawa, S. et al. Comprehensives for guilty feelings in young adults neural network. Neuroimage 105, 248-256, https://doi. org/10.1016/j.neuroimage.2014.11.004 (2015).

8. Wagner, D. D., Haxby, J. V. \& Heatherton, T. F. The representation of self and person knowledge in the medial prefrontal cortex. Wiley interdisciplinary reviews. Cognitive science 3, 451-470, https://doi.org/10.1002/wcs.1183 (2012).

9. Sugiura, M. Associative account of self-cognition: extended forward model and multi-layer structure. Front Hum Neurosci 7, 535, https://doi.org/10.3389/fnhum.2013.00535 (2013).

10. Rolls, E. T. The orbitofrontal cortex. Philosophical transactions of the Royal Society of London. Series B, Biological sciences 351, 14331443; discussion 1443-1434, https://doi.org/10.1098/rstb.1996.0128 (1996).

11. Gillett, G. \& Franz, E. Evolutionary neurology, responsive equilibrium, and the moral brain. Consciousness and cognition 45, 245-250, https://doi.org/10.1016/j.concog.2014.09.011 (2016).

12. Nakagawa, S. et al. Mean diffusivity related to collectivism among university students in Japan. Scientific reports 9, 1338, https://doi. org/10.1038/s41598-018-37995-5 (2019).

13. Lebel, C., Treit, S. \& Beaulieu, C. A review of diffusion MRI of typical white matter development from early childhood to young adulthood. NMR in biomedicine, https://doi.org/10.1002/nbm.3778 (2017).

14. Takeuchi, H. et al. Effect of the interaction between BDNF Val66Met polymorphism and daily physical activity on mean diffusivity. Brain imaging and behavior, https://doi.org/10.1007/s11682-018-0025-8 (2019).

15. Bybee, J. The Emergence of Gender Differencws in Guilt during Adolescence. Guilt and children. 5, 113-125 (Elsevier, 1998).

16. Tangney, J. P. Conceptual and methodological issues in the assessment of shame and guilt. Behaviour research and therapy 34, 741-754 (1996).

17. Roux, F. E., Djidjeli, I. \& Durand, J. B. Functional architecture of the somatosensory homunculus detected by electrostimulation. The Journal of physiology 596, 941-956, https://doi.org/10.1113/jp275243 (2018).

18. Schaefer, M., Rotte, M., Heinze, H. J. \& Denke, C. Dirty deeds and dirty bodies: Embodiment of the Macbeth effect is mapped topographically onto the somatosensory cortex. Scientific reports 5, 18051, https://doi.org/10.1038/srep18051 (2015).

19. Denke, C., Rotte, M., Heinze, H. J. \& Schaefer, M. Lying and the Subsequent Desire for Toothpaste: Activity in the Somatosensory Cortex Predicts Embodiment of the Moral-Purity Metaphor. Cerebral cortex 26, 477-484, https://doi.org/10.1093/cercor/bhul70 (2016).

20. Zhong, C. B. \& Liljenquist, K. Washing away your sins: threatened morality and physical cleansing. Science 313, 1451-1452, https:// doi.org/10.1126/science.1130726 (2006).

21. Damasio, A. R. The somatic marker hypothesis and the possible functions of the prefrontal cortex. Philosophical transactions of the Royal Society of London. Series B, Biological sciences 351, 1413-1420, https://doi.org/10.1098/rstb.1996.0125 (1996).

22. Schmithorst, V. J., Wilke, M., Dardzinski, B. J. \& Holland, S. K. Correlation of white matter diffusivity and anisotropy with age during childhood and adolescence: a cross-sectional diffusion-tensor MR imaging study. Radiology 222, 212-218, https://doi.org/10.1148/ radiol.2221010626 (2002)

23. Takeuchi, H. et al. Impact of videogame play on the brain's microstructural properties: cross-sectional and longitudinal analyses. Molecular psychiatry 21, 1781-1789, https://doi.org/10.1038/mp.2015.193 (2016).

24. Lebel, C. et al. Diffusion tensor imaging of white matter tract evolution over the lifespan. Neuroimage 60, 340-352 (2012).

25. Raichle, M. E. et al. A default mode of brain function. Proc Natl Acad Sci USA 98, 676-682, https://doi.org/10.1073/pnas.98.2.676 (2001).

26. Davey, C. G., Pujol, J. \& Harrison, B. J. Mapping the self in the brain's default mode network. NeuroImage 132, 390-397, https://doi. org/10.1016/j.neuroimage.2016.02.022 (2016).

27. Molnar-Szakacs, I. \& Uddin, L. Q. Self-processing and the default mode network: interactions with the mirror neuron system. Front Hum Neurosci 7, 571, https://doi.org/10.3389/fnhum.2013.00571 (2013).

28. van der Meer, L., Costafreda, S., Aleman, A. \& David, A. S. Self-reflection and the brain: a theoretical review and meta-analysis of neuroimaging studies with implications for schizophrenia. Neurosci Biobehav Rev 34, 935-946, https://doi.org/10.1016/j. neubiorev.2009.12.004 (2010)

29. Jankowski, K. F. \& Takahashi, H. Cognitive neuroscience of social emotions and implications for psychopathology: examining embarrassment, guilt, envy, and schadenfreude. Psychiatry and clinical neurosciences 68,319-336, https://doi.org/10.1111/pcn.12182 (2014).

30. Mancini, A. \& Mancini, F. Do not play God: contrasting effects of deontological guilt and pride on decision-making. Front Psychol 6, 1251, https://doi.org/10.3389/fpsyg.2015.01251 (2015).

31. Buckholtz, J. W. et al. The neural correlates of third-party punishment. Neuron 60, 930-940, https://doi.org/10.1016/j. neuron.2008.10.016 (2008).

32. Sobhani, M. \& Bechara, A. A somatic marker perspective of immoral and corrupt behavior. Social neuroscience 6, 640-652, https:// doi.org/10.1080/17470919.2011.605592 (2011).

33. Bechara, A., Damasio, H., Tranel, D. \& Damasio, A. R. The Iowa Gambling Task and the somatic marker hypothesis: some questions and answers. Trends in cognitive sciences 9, 159-162, https://doi.org/10.1016/j.tics.2005.02.002 (2005).

34. Hansel, A. \& von Kanel, R. The ventro-medial prefrontal cortex: a major link between the autonomic nervous system, regulation of emotion, and stress reactivity? BioPsychoSocial medicine 2, 21, https://doi.org/10.1186/1751-0759-2-21 (2008).

35. Kahane, G. et al. The neural basis of intuitive and counterintuitive moral judgment. Social cognitive and affective neuroscience 7 , 393-402, https://doi.org/10.1093/scan/nsr005 (2012).

36. Pujol, J. et al. Posterior cingulate activation during moral dilemma in adolescents. Human brain mapping 29, 910-921, https://doi. org/10.1002/hbm.20436 (2008).

37. Takeuchi, H. et al. Mean diffusivity of globus pallidus associated with verbal creativity measured by divergent thinking and creativity-related temperaments in young healthy adults. Human brain mapping 36, 1808-1827, https://doi.org/10.1002/hbm.22739 (2015).

38. Basile, B. et al. Deontological and altruistic guilt: evidence for distinct neurobiological substrates. Human brain mapping 32, 229-239, https://doi.org/10.1002/hbm.21009 (2011).

39. Hsu, M., Anen, C. \& Quartz, S. R. The right and the good: distributive justice and neural encoding of equity and efficiency. Science 320, 1092-1095, https://doi.org/10.1126/science.1153651 (2008).

40. Igelstrom, K. M. \& Graziano, M. S. A. The inferior parietal lobule and temporoparietal junction: A network perspective. Neuropsychologia 105, 70-83, https://doi.org/10.1016/j.neuropsychologia.2017.01.001 (2017).

41. Mancini, F. \& Gangemi, A. Deontological guilt and obsessive compulsive disorder. Journal of Behavior Therapy and Experimental Psychiatry 49, 157-163, https://doi.org/10.1016/j.jbtep.2015.05.003 (2015).

42. Tangney, J. P. Assessing individual differences in proneness to shame and guilt: development of the Self-Conscious Affect and Attribution Inventory. J Pers Soc Psychol 59, 102-111 (1990).

43. Else-Quest, N. M., Higgins, A., Allison, C. \& Morton, L. C. Gender differences in self-conscious emotional experience: a metaanalysis. Psychological bulletin 138, 947-981, https://doi.org/10.1037/a0027930 (2012).

44. Michl, P. et al. Neurobiological underpinnings of shame and guilt: a pilot fMRI study. Social cognitive and affective neuroscience 9 , 150-157, https://doi.org/10.1093/scan/nss114 (2014).

45. Jaffee, S. \& Hyde, J. S. Gender differences in moral orientation: a meta-analysis. Psychological bulletin 126, 703-726 (2000). 
46. Ishikawa, T. \& Uchiyama, I. Relations of empathy and social responsibility to guilt feelings among undergraduate students. Perceptual and motor skills $91,1127-1133$ (2000).

47. Takeuchi, H. et al. White matter structures associated with empathizing and systemizing in young adults. Neuroimage $77,222-236$, https://doi.org/10.1016/j.neuroimage.2013.04.004 (2013)

48. Nakagawa, S. et al. Lenticular nucleus correlates of general self-efficacy in young adults. Brain structure \& function 222, 3309-3318, https://doi.org/10.1007/s00429-017-1406-2 (2017).

49. Nakagawa, S. et al. Basal ganglia correlates of fatigue in young adults. Scientific reports 6, 21386, https://doi.org/10.1038/srep21386 (2016).

50. Takeuchi, H. et al. Degree centrality and fractional amplitude of low-frequency oscillations associated with Stroop interference. Neuroimage 119, 197-209 (2015).

51. Nakagawa, S. et al. Sex-Related Differences in the Effects of Sleep Habits on Verbal and Visuospatial Working. Memory. Frontiers in psychology 7, 1128, https://doi.org/10.3389/fpsyg.2016.01128 (2016).

52. Ishikawa, T. \& Uchiyama, I. The Relations of Empathy and Role-Taking Ability to Guilt Feelings in Adolescence. The Japanese Journal of Developmental Psychology 13, 12-19 (2002).

53. Raven, J. Manual for Raven's progressive matrices and vocabulary scales. (Oxford Psychologists Press 1998).

54. Colom, R., Jung, R. E. \& Haier, R. J. Distributed brain sites for the g-factor of intelligence. Neuroimage 31, 1359-1365 (2006).

55. Haier, R. J., Jung, R. E., Yeo, R. A., Head, K. \& Alkire, M. T. Structural brain variation and general intelligence. Neuroimage 23, 425-433, https://doi.org/10.1016/j.neuroimage.2004.04.025 (2004).

56. Narr, K. L. et al. Relationships between IQ and regional cortical gray matter thickness in healthy adults. Cerebral Cortex 17, 2163-2171 (2007).

57. Takeuchi, H. et al. Training of Working Memory Impacts Structural Connectivity. J. Neurosci. 30, 3297-3303 (2010).

58. Hashimoto, T. et al. Neuroanatomical correlates of the sense of control: Gray and white matter volumes associated with an internal locus of control. Neuroimage 119, 146-151 (2015).

59. Ashburner, J. \& Friston, K. J. Voxel-based morphometry-the methods. Neuroimage 11, 805-821 (2000).

60. Takeuchi, H. et al. The structure of the amygdala associates with human sexual permissiveness: Evidence from voxel-based morphometry. Hum. Brain Mapp. 36, 440-448 (2015).

61. Le Bihan, D. et al. Diffusion tensor imaging: concepts and applications. Journal of Magnetic Resonance Imaging 13, 534-546 (2001).

62. Smith, S. M. et al. Tract-based spatial statistics: voxelwise analysis of multi-subject diffusion data. NeuroImage 31, 1487-1505, https://doi.org/10.1016/j.neuroimage.2006.02.024 (2006).

63. Bach, M. et al. Methodological considerations on tract-based spatial statistics (TBSS). NeuroImage 100, 358-369 (2014).

64. Smith, S. M. \& Nichols, T. E. Threshold-free cluster enhancement: addressing problems of smoothing, threshold dependence and localisation in cluster inference. Neuroimage 44, 83-98, https://doi.org/10.1016/j.neuroimage.2008.03.061 (2009).

\section{Acknowledgements}

We respectfully thank Yuki Yamada for operating the MRI scanner and Haruka Nouchi for administering the psychological tests. Furthermore, we would like to thank the participants in the study, the other individuals who administered psychological tests, and our colleagues in the Institute of Development, Ageing and Cancer at Tohoku University for their support. Dr. H.T. was supported by JST/RISTEX, JST/CREST, a Grant-in-Aid for Young Scientists (B) (KAKENHI 23700306), and a Grant-in-Aid for Young Scientists (A) (KAKENHI 25700012) from the Ministry of Education, Culture, Sports, Science, and Technology, and Health Science Center Foundation. Dr. S.N. was financially supported by the Division of Psychiatry, Tohoku Medical and Pharmaceutical University. We would like to thank Editage (www.editage.jp) for English language editing.

\section{Author Contributions}

S.N., H.T., Y.T. and R.K. designed the study. S.N., H.T., R.N., Y.K., T.S., T.M., A.S., K.I., R.Y., Y.Y., S.H., T.A., C.M.M., D.M., K.S., H.J. and Y.S. collected the data. S.N. and H.T. analyzed the data and prepared the manuscript. All authors reviewed the manuscript.

Additional Information

Competing Interests: The authors declare no competing interests.

Publisher's note: Springer Nature remains neutral with regard to jurisdictional claims in published maps and institutional affiliations.

(c) (i) Open Access This article is licensed under a Creative Commons Attribution 4.0 International

License, which permits use, sharing, adaptation, distribution and reproduction in any medium or format, as long as you give appropriate credit to the original author(s) and the source, provide a link to the Creative Commons license, and indicate if changes were made. The images or other third party material in this article are included in the article's Creative Commons license, unless indicated otherwise in a credit line to the material. If material is not included in the article's Creative Commons license and your intended use is not permitted by statutory regulation or exceeds the permitted use, you will need to obtain permission directly from the copyright holder. To view a copy of this license, visit http://creativecommons.org/licenses/by/4.0/.

(C) The Author(s) 2019 